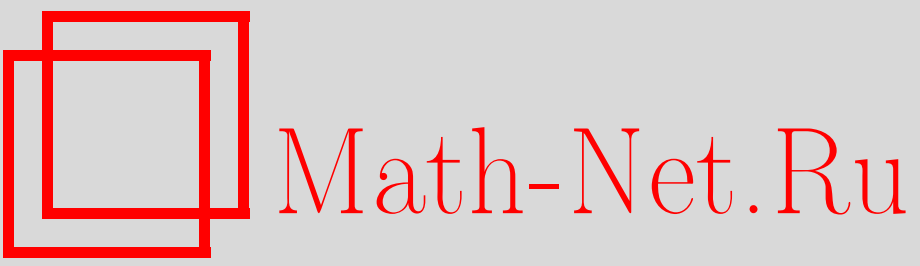

М. М. Анзин, О плотности решетчатого покрытия для $n=13$ и $n=15$, Матем. заметки, 2006, том 79, выпуск 5, 781-784

DOI: https://doi.org/10.4213/mzm2750

Использование Общероссийского математического портала Math-Net.Ru подразумевает, что вы прочитали и согласны с пользовательским соглашением

http: //www.mathnet.ru/rus/agreement

Параметры загрузки:

IP : 54.157 .27 .8

26 апреля 2023 г., 12:28:37 


\section{О ПЛОТНОСТИ РЕШЕТЧАТОГО ПОКРЫТИЯ ДЛЯ $n=13$ И $n=15$}

\section{М. М. Анзин}

1. Настоящая работа является продолжением работ [1], [2] и посвящена задаче о наименее плотных решетчатых покрытиях (см. [3]), которая состоит в отыскании для каждой размерности $n$ такой решетки $\Gamma_{n}$, которая дает наименьшее значение плотности $\theta_{n}(\Gamma)$ решетчатого покрытия евклидова пространства $\mathbb{E}^{n}$ равными шарами.

Как и в $[1],[2]$ мы сводим исследование функции $\theta_{n}(\Gamma)$ к исследованию функции $\eta_{n}(\Gamma)$ :

$$
\eta_{n}(\Gamma)=\eta_{n}\left(f_{\Gamma}\right)=\frac{D^{2}}{\sqrt[n]{\operatorname{det} f_{\Gamma}}}=\frac{(2 R)^{2}}{\sqrt[n]{\operatorname{det} f_{\Gamma}}}
$$

где $D=D(\Gamma)=2 R$ - диаметр шара покрытия, $\operatorname{det} f_{\Gamma}$ - определитель матрицы положительной квадратичной формы $f_{\Gamma}$, отвечаюшей некоторому основному реперу решетки $\Gamma$. Очевидно, что $\eta_{n}(\Gamma)=4\left(\theta_{n}(\Gamma) / \Omega_{n}\right)^{2 / n}$, где $\Omega_{n}$ - объем $n$-мерного шара единичного радиуса.

Задача о наименее плотных решетчатых покрытиях полностью решена только для $n=2,3,4,5$ (см. [3]). Для других $n$ известны только оценки. Существует гипотеза, что решетка Лича $\Lambda_{24}$ дает абсолютный минимум функции $\eta_{n}(\Gamma)$ для $n=24: \eta_{24}\left(\Lambda_{24}\right)=8$. Оценки функции $\eta_{n}(\Gamma)$, лучшие (меньшие), чем $\eta_{n}\left(A_{n}^{*}\right)=n(n+2) \sqrt[n]{n+1} /(3 n+3)$, были найдены для всех размерностей $n>24$ в работах [4] и [5]. Для $5<n<24$ такие оценки получены только для: $n=6$-в [6], $n=9-$ в [7], $n=11,14-$ в [1], [2] $n=12-$ в [8], $n=22,23-$ в [9]. Настоящая работа посвящена подробному изложению аналогичного результата для $n=13$ и $n=15$, анонсированного в [10].

ОСНовНАЯ ТЕОРЕМА. Имеют место соотношения

$$
\begin{aligned}
& \eta_{13} \leqslant \eta_{13}\left(A_{13}^{7}\right)=\frac{248}{49} \sqrt[13]{\frac{7}{2}}=5,573 \cdots<\eta_{13}\left(A_{13}^{*}\right)=5,687 \ldots \\
& \eta_{15} \leqslant \eta_{15}\left(A_{15}^{8}\right)=\frac{23}{4} \sqrt[15]{4}=6,306 \cdots<\eta_{15}\left(A_{15}^{*}\right)=6,391 \ldots
\end{aligned}
$$

əде $\eta_{n}=\inf _{\Gamma \subset \mathbb{E}^{n}} \eta_{n}(\Gamma)=\min _{\Gamma \subset \mathbb{E}^{n}} \eta_{n}(\Gamma), A_{13}^{7} u A_{15}^{8}-$ решеткu Kокстера (см. [11]).

В пункте 3 мы приводим таблицы 1 и 2 , информация в которых, фактически, и является доказательством основной теоремы. В свою очередь, таблицы 1 и 2 получены на основе полного описания строения $L$-разбиения решеток $A_{13}^{7}$ и $A_{15}^{8}$ (см. пункт 2, утверждения 1 и 2 ).

2. Как и в работах [1], [2] мы сводим исследование форм Кокстера $A_{13}^{7}$ и $A_{15}^{8}$ к исследованию эквивалентных им форм $\varphi^{(13,7)} \sim 2 A_{13}^{7}$ и $\varphi^{(15,8)} \sim 2 A_{15}^{8}$ :

$$
\varphi^{(n, r)}(\mathbf{x})=q_{1}^{2}(\mathbf{x})+q_{2}^{2}(\mathbf{x})+\cdots+q_{n+1}^{2}(\mathbf{x})
$$

где $q_{i}(\mathbf{x})=x_{i}+x_{n} / r, i=1, \ldots, n-1 ; q_{n}(\mathbf{x})=x_{n} / r ; q_{n+1}(\mathbf{x})=-x_{1}-\cdots-x_{n-1}-n x_{n} / r$.

формы $\varphi^{(13,7)}$ и $\varphi^{(15,8)}$ имеют нижеследуюшие характеристики. Арифметический минимум $\min \varphi^{(13,7)}=12 / 7, \min \varphi^{(15,8)}=7 / 4$; число пар минимальных векторов $s\left(\varphi^{(13,7)}\right)=91$, $s\left(\varphi^{(15,8)}\right)=120 ;$ определитель $\operatorname{det} \varphi^{(13,7)}=2 / 7, \operatorname{det} \varphi^{(15,8)}=1 / 4$. В обоих случаях порядок группы автоморфизмов

$$
|G|=\left|G\left(\varphi^{(n, r)}\right)\right|=2(n+1) !
$$

УТВЕРЖДЕНИЕ 1. L-разбиение решетки $\varphi^{(13,7)}$ образовано многогранниками, конгруентными 10 попарно неәквивалентным L-многогранникам, характеристики которых указаны в табличе 1. Максимальное значение радиуса шара, описанного вокруг L-многогранника, достигается на многогранниках класса $L_{6}$ и квадрат радиуса решетчатого покрытия равен $R^{2}\left(\varphi^{(13,7)}\right)=62 / 49$. Тем самым, $\eta_{13}\left(\varphi^{(13,7)}\right)=248 / 49 \cdot \sqrt[13]{7 / 2}=5,573 \ldots$.

Работа выполнена при финансовой поддержке INTAS, проект 03-51-5070. 
ТАБлица 1. Данные для формулы объемов решетки $\varphi^{(13,7)}$

\begin{tabular}{|c|c|c|c|c|c|c|}
\hline $\mathbf{L}_{\alpha}$ & $R^{2}$ & $\sharp$ & Vol & $\mathscr{A}_{\beta}$ & Центр описанного шара $\mathscr{A}_{\beta}$ & $\left|\operatorname{St}\left(\mathscr{A}_{\beta}\right)\right|$ \\
\hline $\mathbf{L}_{7}$ & $1 \frac{3}{14}$ & 14 & 1 & $\mathscr{A}_{1}$ & $\left(\frac{-5}{14}\right)^{2}\left(\frac{5}{14}\right)^{2}\left(\frac{-3}{14}\right)^{2}\left(\frac{3}{14}\right)^{2}\left(\frac{-1}{14}\right)^{2}\left(\frac{1}{14}\right)^{2}\left(\frac{-1}{2}\right)^{1}\left(\frac{1}{2}\right)^{1}$ & 128 \\
\hline \multirow{5}{*}{$\mathbf{L}_{5}$} & $1 \frac{101}{441}$ & 14 & 3 & $\overline{A_{2}}$ & $\left(\frac{-5}{21}\right)^{4}(0)^{3}\left(\frac{8}{21}\right)^{3}\left(\frac{2}{7}\right)^{2}\left(\frac{-1}{7}\right)^{1}\left(\frac{-13}{21}\right)^{1}$ & 1728 \\
\hline & & & & $\mathscr{A}_{3}$ & $\left(\frac{5}{21}\right)^{4}\left(\frac{-8}{21}\right)^{3}\left(\frac{-1}{7}\right)^{3}\left(\frac{1}{7}\right)^{2}\left(\frac{-2}{7}\right)^{1}\left(\frac{13}{21}\right)^{1}$ & 1728 \\
\hline & & & & $\mathscr{A}_{4}$ & $\left(\frac{-4}{21}\right)^{4}\left(\frac{4}{21}\right)^{4}\left(\frac{-2}{7}\right)^{2}\left(\frac{3}{7}\right)^{2}\left(\frac{2}{7}\right)^{1}\left(\frac{-4}{7}\right)^{1}$ & 2304 \\
\hline & & & & $\mathscr{A}_{5}$ & $\left(\frac{-1}{3}\right)^{4}\left(\frac{1}{21}\right)^{4}\left(\frac{2}{7}\right)^{3}\left(\frac{-3}{7}\right)^{1}\left(\frac{4}{7}\right)^{1}\left(\frac{1}{7}\right)^{1}$ & 3456 \\
\hline & & & & $\mathscr{A}_{6}$ & $\left(\frac{-1}{21}\right)^{4}\left(\frac{1}{3}\right)^{4}\left(\frac{-3}{7}\right)^{3}\left(\frac{-1}{7}\right)^{2}\left(\frac{3}{7}\right)^{1}$ & 6912 \\
\hline \multirow[t]{3}{*}{$\mathbf{L}_{8}$} & $1 \frac{205}{882}$ & 14 & 3 & $\mathscr{A}_{7}$ & $\left(\frac{11}{42}\right)^{4}\left(\frac{-17}{42}\right)^{3}\left(\frac{-3}{14}\right)^{2}\left(\frac{-1}{14}\right)^{2}\left(\frac{1}{14}\right)^{2}\left(\frac{25}{42}\right)^{1}$ & 1152 \\
\hline & & & & $\mathscr{A}_{8}$ & $\left(\frac{-13}{42}\right)^{4}\left(\frac{1}{42}\right)^{4}\left(\frac{3}{14}\right)^{2}\left(\frac{5}{14}\right)^{2}\left(\frac{-1}{2}\right)^{1}\left(\frac{1}{2}\right)^{1}$ & 2304 \\
\hline & & & & $\mathscr{A}_{9}$ & $\left(\frac{-1}{6}\right)^{4}\left(\frac{1}{6}\right)^{4}\left(\frac{-5}{14}\right)^{2}\left(\frac{5}{14}\right)^{2}\left(\frac{-1}{2}\right)^{1}\left(\frac{1}{2}\right)^{1}$ & 4608 \\
\hline \multirow[t]{2}{*}{$\mathbf{L}_{2}$} & $1 \frac{509}{2450}$ & 14 & 5 & $\mathscr{A}_{10}$ & $\left(\frac{13}{70}\right)^{6}\left(\frac{-23}{70}\right)^{5}\left(\frac{-1}{14}\right)^{2}\left(\frac{47}{70}\right)^{1}$ & 172800 \\
\hline & & & & $\mathscr{A}_{11}$ & $\left(\frac{-17}{70}\right)^{6}\left(\frac{17}{70}\right)^{6}\left(\frac{-1}{2}\right)^{1}\left(\frac{1}{2}\right)^{1}$ & 1036800 \\
\hline \multirow[t]{3}{*}{$\mathbf{L}_{3}$} & $1 \frac{571}{2401}$ & 14 & 7 & $\overline{\mathscr{A}_{12}}$ & $\left(\frac{-10}{49}\right)^{5}\left(\frac{17}{49}\right)^{5}\left(\frac{-1}{49}\right)^{3}\left(\frac{-32}{49}\right)^{1}$ & 86400 \\
\hline & & & & $\mathscr{A}_{13}$ & $\left(\frac{10}{49}\right)^{6}\left(\frac{-17}{49}\right)^{4}\left(\frac{-8}{49}\right)^{3}\left(\frac{32}{49}\right)^{1}$ & 103680 \\
\hline & & & & $\mathscr{A}_{14}$ & $\left(\frac{-11}{49}\right)^{6}\left(\frac{11}{49}\right)^{5}\left(\frac{20}{49}\right)^{2}\left(\frac{-29}{49}\right)^{1}$ & 172800 \\
\hline \multirow[t]{2}{*}{$\mathbf{L}_{1}$} & $1 \frac{1}{7}$ & 15 & 21 & $\mathscr{A}_{15}$ & $\left(\frac{-1}{7}\right)^{7}\left(\frac{2}{7}\right)^{6}\left(\frac{-5}{7}\right)^{1}$ & 3628800 \\
\hline & & & & 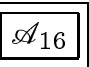 & $\left(\frac{-2}{7}\right)^{7}\left(\frac{2}{7}\right)^{7}$ & 50803200 \\
\hline \multirow[t]{5}{*}{$\mathbf{L}_{4}$} & $1 \frac{23}{98}$ & 15 & 24 & $\overline{\mathscr{A}_{17}}$ & $\left(\frac{-3}{14}\right)^{5}\left(\frac{5}{14}\right)^{4}(0)^{3}\left(\frac{-9}{14}\right)^{1}\left(\frac{2}{7}\right)^{1}$ & 17280 \\
\hline & & & & $\mathscr{A}_{18}$ & $\left(\frac{3}{14}\right)^{5}\left(\frac{-5}{14}\right)^{4}\left(\frac{-1}{7}\right)^{3}\left(\frac{1}{7}\right)^{1}\left(\frac{9}{14}\right)^{1}$ & 17280 \\
\hline & & & & $\mathscr{A}_{19}$ & $\left(\frac{-3}{14}\right)^{5}\left(\frac{3}{14}\right)^{5}\left(\frac{3}{7}\right)^{2}\left(\frac{-4}{7}\right)^{1}\left(\frac{-2}{7}\right)^{1}$ & 28800 \\
\hline & & & & $\mathscr{A}_{20}$ & $\left(\frac{-5}{14}\right)^{5}\left(\frac{1}{14}\right)^{5}\left(\frac{2}{7}\right)^{3}\left(\frac{4}{7}\right)^{1}$ & 86400 \\
\hline & & & & $\mathscr{A}_{21}$ & $\left(\frac{-1}{14}\right)^{5}\left(\frac{5}{14}\right)^{5}\left(\frac{-3}{7}\right)^{3}\left(\frac{-1}{7}\right)^{1}$ & 86400 \\
\hline \multirow[t]{2}{*}{$\mathbf{L}_{9}$} & $1 \frac{25}{98}$ & 20 & 346 & $\mathscr{A}_{22}$ & $\left(\frac{5}{14}\right)^{5}\left(\frac{-3}{14}\right)^{4}\left(\frac{-1}{14}\right)^{4}\left(\frac{-9}{14}\right)^{1}$ & 69120 \\
\hline & & & & $\mathscr{A}_{23}$ & $\left(\frac{-3}{14}\right)^{6}\left(\frac{3}{14}\right)^{4}\left(\frac{5}{14}\right)^{3}\left(\frac{-9}{14}\right)^{1}$ & 103680 \\
\hline \multirow[t]{4}{*}{$\mathbf{L}_{6}$} & $1 \frac{13}{49}$ & 23 & 1397 & $\mathscr{A}_{24}$ & $\left(\frac{-2}{7}\right)^{4}(0)^{3}\left(\frac{1}{7}\right)^{3}\left(\frac{3}{7}\right)^{3}\left(\frac{-4}{7}\right)^{1}$ & 5184 \\
\hline & & & & $\mathscr{A}_{25}$ & $\left(\frac{-1}{7}\right)^{4}\left(\frac{1}{7}\right)^{3}\left(\frac{2}{7}\right)^{3}\left(\frac{-3}{7}\right)^{3}\left(\frac{4}{7}\right)^{1}$ & 5184 \\
\hline & & & & $\mathscr{A}_{26}$ & $\left(\frac{-2}{7}\right)^{4}(0)^{4}\left(\frac{2}{7}\right)^{3}\left(\frac{3}{7}\right)^{2}\left(\frac{-4}{7}\right)^{1}$ & 6912 \\
\hline & & & & $\mathscr{A}_{27}$ & $\left(\frac{-1}{7}\right)^{4}\left(\frac{1}{7}\right)^{4}\left(\frac{3}{7}\right)^{3}\left(\frac{-3}{7}\right)^{3}$ & 41472 \\
\hline $\mathbf{L}_{0}$ & $\frac{13}{14}$ & 28 & 12012 & $\mathscr{A}_{28}$ & $\left(\frac{1}{14}\right)^{13}\left(\frac{-13}{14}\right)^{1}$ & 6227020800 \\
\hline
\end{tabular}

УТВЕРЖДЕнИЕ 2. L-разбиение решетки $\varphi^{(15,8)}$ образовано многогранниками, конгруентными 10 попарно неәквивалентным $L$-многогранникам, характеристики которых указаны в таблице 2. Максимальное значение радиуса иара, описанного вокруг L-многогранника, достигается на многогранниках двух классов $L_{1}$ и $L_{2}$, квадрат радиуса решетчатого покрытия равен $R^{2}\left(\varphi^{(15,8)}\right)=23 / 16$. Тем самым,$\eta_{15}\left(\varphi^{(15,8)}\right)=23 / 4 \cdot \sqrt[15]{4}=$ $6,306 \ldots$ 
ТАБлица 2. Данные для формулы объемов решетки $\varphi^{(15,8)}$

\begin{tabular}{|c|c|c|c|c|c|c|}
\hline $\mathbf{L}_{\alpha}$ & $R^{2}$ & $\sharp$ & Vol & $\mathscr{A}_{\beta}$ & Центр описанного шара $\mathscr{A}_{\beta}$ & $\left|\operatorname{St}\left(\mathscr{A}_{\beta}\right)\right|$ \\
\hline \multirow[t]{3}{*}{$\mathbf{L}_{4}$} & $1 \frac{115}{288}$ & 16 & 6 & & $\left(\frac{-1}{12}\right)^{4}\left(\frac{5}{24}\right)^{3}\left(\frac{7}{24}\right)^{3}\left(\frac{-5}{12}\right)^{3}\left(\frac{-1}{4}\right)^{2}\left(\frac{7}{12}\right)^{1}$ & 10368 \\
\hline & & & & $\mathscr{A}_{2}$ & $\left(\frac{-7}{24}\right)^{4}\left(\frac{1}{24}\right)^{4}\left(\frac{1}{3}\right)^{3}\left(\frac{-1}{8}\right)^{2}\left(\frac{5}{12}\right)^{2}\left(\frac{-7}{12}\right)^{1}$ & 13824 \\
\hline & & & & $\mathscr{A}_{3}$ & $\left(\frac{-1}{3}\right)^{4}\left(\frac{1}{3}\right)^{4}\left(\frac{-1}{24}\right)^{3}\left(\frac{1}{24}\right)^{3}\left(\frac{-1}{2}\right)^{1}\left(\frac{1}{2}\right)^{1}$ & 41472 \\
\hline \multirow[t]{3}{*}{$\mathbf{L}_{8}$} & $1 \frac{49}{128}$ & 18 & 92 & & $\left(\frac{1}{4}\right)^{5}\left(\frac{-3}{8}\right)^{4}\left(\frac{-3}{16}\right)^{3}\left(\frac{1}{16}\right)^{3}\left(\frac{5}{8}\right)^{1}$ & 103680 \\
\hline & & & & $\mathscr{A}_{5}$ & $\left(\frac{-1}{4}\right)^{5}\left(\frac{1}{8}\right)^{5}\left(\frac{5}{16}\right)^{3}\left(\frac{-7}{16}\right)^{2}\left(\frac{9}{16}\right)^{1}$ & 172800 \\
\hline & & & & & $\left(\frac{-3}{8}\right)^{5}(0)^{5}\left(\frac{3}{16}\right)^{3}\left(\frac{7}{16}\right)^{3}$ & 518400 \\
\hline \multirow[t]{3}{*}{$\mathbf{L}_{7}$} & $1 \frac{59}{144}$ & 20 & 162 & $\mathscr{A}_{7}$ & $\left(\frac{-11}{48}\right)^{4}\left(\frac{5}{48}\right)^{4}\left(\frac{7}{16}\right)^{3}\left(\frac{-5}{16}\right)^{2}\left(\frac{3}{16}\right)^{2}\left(\frac{-9}{16}\right)^{1}$ & 13824 \\
\hline & & & & & $\left(\frac{-1}{16}\right)^{4}\left(\frac{13}{48}\right)^{4}\left(\frac{-19}{48}\right)^{3}\left(\frac{-5}{16}\right)^{2}\left(\frac{3}{16}\right)^{2}\left(\frac{29}{48}\right)^{1}$ & 13824 \\
\hline & & & & & $\left(\frac{-17}{48}\right)^{4}\left(\frac{-1}{48}\right)^{4}\left(\frac{5}{16}\right)^{4}\left(\frac{1}{16}\right)^{2}\left(\frac{-7}{16}\right)^{1}\left(\frac{9}{16}\right)^{1}$ & 27648 \\
\hline \multirow[t]{3}{*}{$\mathbf{L}_{6}$} & $1 \frac{53}{128}$ & 20 & 192 & $\mathscr{A}_{10}$ & $\left(\frac{1}{4}\right)^{5}\left(\frac{-3}{8}\right)^{4}\left(\frac{-1}{16}\right)^{4}\left(\frac{-5}{16}\right)^{1}\left(\frac{3}{16}\right)^{1}\left(\frac{5}{8}\right)^{1}$ & 69120 \\
\hline & & & & $\mathscr{A}_{11}$ & $\left(\frac{-1}{8}\right)^{5}\left(\frac{1}{4}\right)^{5}\left(\frac{-7}{16}\right)^{3}\left(\frac{-3}{16}\right)^{1}\left(\frac{5}{16}\right)^{1}\left(\frac{9}{16}\right)^{1}$ & 86400 \\
\hline & & & & $\mathscr{A}_{12}$ & $(0)^{5}\left(\frac{3}{8}\right)^{5}\left(\frac{-5}{16}\right)^{4}\left(\frac{-9}{16}\right)^{1}\left(\frac{-1}{16}\right)^{1}$ & 345600 \\
\hline \multirow[t]{2}{*}{$\mathbf{L}_{9}$} & $1 \frac{167}{400}$ & 20 & 270 & $\mathscr{A}_{13}$ & $\left(\frac{19}{80}\right)^{6}\left(\frac{-29}{80}\right)^{5}\left(\frac{-1}{16}\right)^{4}\left(\frac{51}{80}\right)^{1}$ & 2073600 \\
\hline & & & & $\mathscr{A}_{14}$ & $\left(\frac{-21}{80}\right)^{6}\left(\frac{11}{80}\right)^{6}\left(\frac{7}{16}\right)^{3}\left(\frac{-9}{16}\right)^{1}$ & 3110400 \\
\hline \multirow[t]{4}{*}{$\mathbf{L}_{3}$} & $1 \frac{13}{32}$ & 22 & 624 & $\mathscr{A}_{15}$ & $\left(\frac{-7}{16}\right)^{3}\left(\frac{-3}{16}\right)^{3}\left(\frac{-1}{16}\right)^{3}\left(\frac{3}{16}\right)^{3}\left(\frac{5}{16}\right)^{3}\left(\frac{9}{16}\right)^{1}$ & 7776 \\
\hline & & & & & $\left(\frac{-1}{16}\right)^{4}\left(\frac{3}{16}\right)^{3}\left(\frac{5}{16}\right)^{3}\left(\frac{-5}{16}\right)^{3}\left(\frac{-7}{16}\right)^{2}\left(\frac{9}{16}\right)^{1}$ & 10368 \\
\hline & & & & $\mathscr{A}_{17}$ & $\left(\frac{5}{16}\right)^{4}\left(\frac{-5}{16}\right)^{3}\left(\frac{-1}{16}\right)^{3}\left(\frac{1}{16}\right)^{3}\left(\frac{-7}{16}\right)^{2}\left(\frac{9}{16}\right)^{1}$ & 10368 \\
\hline & & & & $\mathscr{A}_{18}$ & $\left(\frac{-3}{16}\right)^{4}\left(\frac{-7}{16}\right)^{3}\left(\frac{1}{16}\right)^{3}\left(\frac{3}{16}\right)^{3}\left(\frac{7}{16}\right)^{3}$ & 31104 \\
\hline \multirow[t]{3}{*}{$\mathbf{L}_{5}$} & $1 \frac{13}{32}$ & 23 & 1620 & $\mathscr{A}_{19}$ & $\left(\frac{-1}{4}\right)^{5}(0)^{5}\left(\frac{3}{8}\right)^{5}\left(\frac{-5}{8}\right)^{1}$ & 1728000 \\
\hline & & & & $\mathscr{A}_{20}$ & $\left(\frac{-1}{4}\right)^{6}\left(\frac{1}{8}\right)^{5}\left(\frac{3}{8}\right)^{4}\left(\frac{-5}{8}\right)^{1}$ & 2073600 \\
\hline & & & & $\mathscr{A}_{21}$ & $(0)^{6}\left(\frac{-3}{8}\right)^{5}\left(\frac{3}{8}\right)^{5}$ & 20736000 \\
\hline $\mathbf{L}_{2}$ & $1 \frac{7}{16}$ & 32 & 50968 & $\mathscr{A}_{22}$ & $\left(\frac{-5}{16}\right)^{4}\left(\frac{-1}{16}\right)^{4}\left(\frac{3}{16}\right)^{4}\left(\frac{7}{16}\right)^{3}\left(\frac{-9}{16}\right)^{1}$ & 82944 \\
\hline $\mathbf{L}_{0}$ & $\frac{15}{16}$ & 32 & 51480 & $\mathscr{A}_{23}$ & $\left(\frac{1}{16}\right)^{15}\left(\frac{-15}{16}\right)^{1}$ & 1307674368000 \\
\hline $\mathbf{L}_{1}$ & $1 \frac{7}{16}$ & 32 & 154440 & $\mathscr{A}_{24}$ & $\left(\frac{-3}{16}\right)^{8}\left(\frac{5}{16}\right)^{7}\left(\frac{-11}{16}\right)^{1}$ & 203212800 \\
\hline
\end{tabular}

3. Далее мы доказываем утверждения 1 и 2 , для чего вначале при помощи (2) - формулы объемов (см. [2]) - проверяем полноту списка $L$-многогранников, указанных в таблицах 1 и 2 . В обоих случаях формула объемов удовлетворена, что говорит о полноте списка. Далее квадрат радиуса покрытия находится как максимальное из значений в графе “ $R^{2}$ ” таблиц 1 и 2 .

Имеем

$$
\sum_{\beta=1}^{28} \frac{\left|G\left(\varphi^{(13,7)}\right)\right| \cdot \operatorname{Vol}_{\text {отн }}\left(\mathbf{L}_{\alpha}\right)}{\left|\operatorname{St}\left(\mathscr{A}_{\beta}\right)\right| \cdot \sharp\left(\mathbf{L}_{\alpha}\right)}=n !=13 \text { ! и } \sum_{\beta=1}^{24} \frac{\left|G\left(\varphi^{(15,8)}\right)\right| \cdot \operatorname{Vol}_{\text {отн }}\left(\mathbf{L}_{\alpha}\right)}{\left|\operatorname{St}\left(\mathscr{A}_{\beta}\right)\right| \cdot \sharp\left(\mathbf{L}_{\alpha}\right)}=n !=15 \text { !. }
$$

Далее основная теорема доказывается на основе утверждений 1 и 2 при помощи формулы (1).

Дадим краткие пояснения к таблицам 1 и 2 (более подробную информацию см. в [2]). В графе "L $\alpha$ " (в обоих случаях $\alpha=0, \ldots, 9)$ указаны обозначения $L$-тел решеток $\varphi^{(13,7)}$ и $\varphi^{(15,8)}$. В графе " $R^{2}$ " - значение $R^{2}\left(\mathbf{L}_{\alpha}\right)$ - квадрата радиуса шара, описанного вокруг $L$-тела $\mathbf{L}_{\alpha}$. В графе "Vol" - относительный объем $\operatorname{Vol}_{\text {отн }}\left(\mathbf{L}_{\alpha}\right) L$-тел. В графе "甘" - число вершин $\sharp\left(\mathbf{L}_{\alpha}\right) L$-тел. В графе 
“ $\mathscr{A}_{\beta}$ " ( $\beta=1, \ldots, 28$ и $\beta=1, \ldots, 24$, соответственно для $n=13$ и $\left.n=15\right)$ - обозначения арифметических типов $L$-тел $\mathbf{L}_{\alpha}(\alpha=0, \ldots, 9)$, входяших в $L$-звезду $\operatorname{Star}(\mathbf{0})$ начальной точки решетки. В графе "Центр описанного шара $\mathscr{A}_{\beta}$ " - степенная запись для арифметического типа центра шара, описанного вокруг $L$-тела, отвечающего $\mathscr{A}_{\beta}$. В графе "| $\operatorname{St}\left(\mathscr{A}_{\beta}\right) \mid$ " - порядок группы стабилизатора для центра описанного шара, отвечаюшего $\mathscr{A}_{\beta}$.

\section{СПИСОК ЦИТИРОВАННОЙ ЛИТЕРАТУРЫ}

1. Анзин М. М. // УМН. 2002. Т. 57. №2. С. 187-188. 2. Анзин М. М. О плотности решетчатого покрытия для $n=11$ и $n=14 / / \mathrm{K} 70$-летию со дня рожд. проф. Сергея Сергеевича Рышкова. Тр. МИАН. 2002. Т. 239. С. 20-51. 3. Рышков С. С., Барановский Е. П. С-типы $n$-мерных решеток и пятимерные примитивные параллелоэдры (с приложением к теории покрытий) // Тр. МИАН, Т. 137. М.: Наука, 1976. 4. Рышков С. С. // Докл. АН СССР. 1967. Т. 175. № 2. C. 303-305. 5. Bambah R. P., Sloane N. J. A. // Acta Arithm. 1982. V. 42. P. 107-109. 6. Vallentin F. Sphere coverings, lattices, and tilings (in Low Dimensions). D. Dissertation. Technische Universität München, 2003. 7. Baranovskii E. P. // Europ. J. Comb. 1994. V. 15. P. 317-323. 8. Анзин М. М. // Материалы VIII Междунар. семинара "Дискретная математика и ее приложения". МГУ. 2004. С. 374-377. 9. Smith W. D. Studies in computational geometry motivated by mesh generation: Ph.D. Diss. Dept. Appl. Math. Princeton Univ., 1988. 10. Анзин M. М. // Алгебра и теория чисел. Тез. докл. V Междунар. конф. Тула. 2003. С. 15-17. 11. Coxeter H. S. M. // Canad. J. Math. 1951. V. 3. P. 391-441.

E-mail: centr_on@mtu-net.ru

Поступило 19.08.2003

Исправленный вариант 10.10 .2005

\section{ОЦЕНКА ПРИБЛИЖЕНИЯ ПЕРИОДИЧЕСКИХ ФУНКЦИЙ СУММАМИ ФУРЬЕ}

\section{О.В. Бесов}

Везде далее $f-2 \pi$-периодическая непрерывная функция, $1 \leqslant p<\infty$,

$$
\begin{gathered}
\omega(f, \delta)=\sup _{|x-y| \leqslant \delta}|f(x)-f(y)|, \\
\omega(f, \delta)_{p}=\sup _{0<h \leqslant \delta}\left\{\int_{0}^{2 \pi}|f(x+h)-f(x)|^{p} d x\right\}^{\frac{1}{p}},
\end{gathered}
$$

$S_{n}(f ; x)$ - сумма Фурье функции $f$.

Теорема. Пусть $f-2 \pi$-периодическая непрерывная функция, $f \not \equiv$ const, $n \in \mathbb{N}, 1 \leqslant$ $p<\infty$. Тогда

$$
\left|f(x)-S_{n}(f, x)\right| \leqslant C \omega\left(f, \frac{1}{n}\right)\left\{1+p \ln \left[1+\frac{n^{\frac{1}{p}} \omega\left(f, \frac{1}{n}\right)_{p}}{\omega\left(f, \frac{1}{n}\right)}\right]\right\},
$$

где $C$ не зависит ни от $f$, ни от $n$, ни от $p$.

Работа выполнена при финансовой поддержке Российского фонда фундаментальных исследований, грант № 05-01-01050, программы "Ведушие научные школы”, проект НШ-1549.2003.1, и программы РАН "Современные проблемы теоретической математики". 